\title{
Perspectives on the treatment of the longitudinal spinal cord injury
}

\section{Thomas Carlstedt*}

Department of Clinical Science and Education, Hand Surgery, Karolinska Institute, Södersjukhuset, Stockholm, Sweden

\section{Edited by:}

Bo-Michael Bellander, Karolinska

University Hospital, Sweden

\section{Reviewed by:}

Per Mattsson, Karolinska University

Hospital, Sweden

Leif A. Havton, University of California

Los Angeles Neurorehabilitation and

Research Center, USA

\section{*Correspondence}

Thomas Carlstedt, Department of Clinical

Science and Education, Hand Surgery,

Karolinska Institute, Södersjukhuset,

SE-11883 Stockholm, Sweden.

e-mail: carlstedt.thomas@

googlemail.com
The current technique for surgical treatment of the longitudinal spinal cord injury has proven to be successful for restoration of some motor function and alleviation of pain. This has been the first step in finding a cure for spinal cord injuries, but so far there are many obstacles still to be overcome. In this particular injury return of function from spinal cord surgery is only partial. Some of the main remaining problems are related to injury-induced neuronal death, direction and specificity of regeneration and muscle, and receptor disintegration from long time denervation. Currently this is a surgical procedure without any adjuvant treatments. In order to gain further functional improvement combinational therapies developed in basic science experiments are essential.

\section{Keywords: nerve plexus, spinal cord injury, root avulsion, spinal cord surgery, functional recovery}

\section{PRESENT AND PAST}

A spinal cord injury that affects mainly the final common motor pathway and the primary sensory trajectories occurs as the spinal nerve roots are torn or avulsed from their medullary attachments. In humans this occurs most frequently in traction injuries to the brachial plexus but also in trauma to the lumbosacral plexus and cauda equina as well as conus medullaris. Root avulsion interrupts the local transverse segmental spinal cord motor and sensory nerve fibers and is a longitudinal spinal cord injury. This injury has a serious effect of the pertinent spinal cord segment with the breakdown of connections and networks and the development of a spinal cord scar. Disintegration of the shorter central part of the sensory axon and most of the motoneuron axon with a dying back into the spinal cord follows. Within a few weeks after such a trauma there will be an increasing death of neurons in the spinal cord, affecting motoneurons, and autonomic neurons in the ventral horn as well as sensory neurons in the dorsal horn. There is, however, very little if any disintegration of the primary sensory neurons in the ganglion in the dorsal root (Carlstedt, 2007).

The functional consequence of such injury is lower motoneuron syndromes, associated with autonomic paralysis including dysfunctional internal organs, limb muscle atrophy, sensory impairment, and chronic pain. These injuries most often occur as a result of road traffic accidents or violent acts. About 1000 patients are affected annually in the UK. Nerve root injury has been associated with an overall poor clinical outcome as a successful surgical repair would require axonal regrowth within the central nervous system as spinal cord regeneration. Today, therefore, most patients with this type of injury are not treated at all or receive non-curative palliative surgery, which do not give the same level of recovery and pain alleviation.

Experimentally, it was obvious that after dorsal root avulsion and repair sensory axons when encounter the astrocyte dominated CNS part of the root are immobilized and seem to be "stabilized", rather than "repelled" (Carlstedt, 1985). It seems as if they stop because of synapse formation which might be more instructive in the inhibition than inhibitory molecules or lack of intrinsic regenerative capacity. Electron microscopical analysis showed that at least some of these axons form nerve terminals exhibiting active zones which contain transmitter substance. This is another way of inhibition than what currently is the concept (He and Koprivica, 2006). There are a number of reports of induced regeneration of dorsal roots by transplantation of glia cells (Kliot et al., 1990), conditioning lesions (Chong et al., 1999), or application of growth factors (Ramer et al., 2002; Wang et al., 2008). None of those strategies have, however, been useful for clinical applications. A type of stem cells, the olfactory ensheathing cells (OEC) when applied to dorsal roots after injury aggregate in the transitional region without entering into the spinal cord and seem to form a scaffold for the regrowing sensory fibers which can cross into the spinal cord. This technique is now just about to be applied clinically for restoration of sensory function after root injury (Li et al., 2004).

The intracellular neurophysiological and staining technique that unequivocally demonstrated that defined injured spinal cord motoneurons after avulsion and replantation of detached ventral roots could regrow new central nervous axons is the golden standard by which any claim of CNS regeneration should be assessed. In those experiments restoration of muscle function by the injured motoneurons could be confirmed (Cullheim et al., 1989). In recent experiments the recovery of autonomic visceral function was demonstrated from the application of this surgical method in conus medullaris and cauda equina injuries as re-innervation of the lower urinary tract occurred (Hoang et al., 2006).

The biological background to such exceptions to the rule of unsuccessful regeneration in the CNS remains partly elusive. 
A human surgical strategy for the repair of avulsed ventral roots and the longitudinal spinal cord injury was developed from the experimental findings. This line of work has been successfully continued in clinical studies and a real translation of laboratory experiments and results to clinical cases was commenced about 20 years ago (Carlstedt et al., 1995; Carlstedt, 2007). By the replantation of avulsed ventral roots or reconnection by means of nerve grafts between the pertinent spinal cord segments and the avulsed ventral roots there was recovery of useful function mainly to proximal limb muscles after brachial as well as lumbosacral plexus injuries. The best results have been noted in a few preadolescent patients where useful hand function was restored from replantation of lower spinal roots to the brachial plexus. Restored locomotor function was described after lumbosacral plexus repair (Carlstedt, 2007).

Neuropathic pain commonly follows avulsion injuries to the brachial plexus as well as cauda equina injuries. Interestingly, there is a significant correlation between pain intensity and the number of avulsed roots. The mechanism behind nerve root avulsion induced pain is not understood but there is a conspicuous alleviation of this pain with recovery of muscle but not sensory function (Berman et al., 1998; Htut et al., 2006). Thus in patients with a complete brachial plexus avulsion injury where replantation has been performed there is in conjunction with restored muscle activity diminished pain (Havton and Carlstedt, 2009). In patients with re-established muscle function throughout the extremity-also in the hand-there is a total loss of pain (Carlstedt et al., 2004). Obviously motor rather than sensory function is of significance. Basic science correlates to this clinical observation has recently been done as ventral root avulsion is capable of eliciting damage that propagates from the ventral to the dorsal horn and can cause degeneration in primary afferent fibers (Bigbee et al., 2008; Havton and Carlstedt, 2009). Interestingly, ventral root implantation protects against afferent degeneration, and reduces the glia response as well as promotes motoneuron survival (Bergerot et al., 2004; Bigbee et al., 2008; Eggers et al., 2008).

With the current surgical technique recovery of sensory function is poor as restoration of primary sensory neuron contacts in the dorsal horn has clinically not been possible. Intriguing is, however, that even without sensory feedback such as proprioception from muscles there was recovery of useful function also in the hand when exclusively motor trajectories were restored from spinal cord implantation. Functional MRI of cortical activities in such a case has been performed (Carlstedt et al., 2009). Not surprisingly it was shown that when the affected hand was used there was bilateral cortical activities which did not happen in the normal situation. This is related to cortical plasticity as there is a bilateral cortical representation for each side but normally the ipsilateral cortex is inhibited through transcallosal connections which in circumstances such as multiple sclerosis and stroke are disinhibited. There was also and more remarkable activity in the sensory cortex when the deafferentated hand was active. This is not an effect of plasticity but most likely represent normality. This finding demonstrates in fact that there is a cortical sensory program that acts independently of afferents and is serving motor commands. This is of conceptual importance and means that proprioception is not obligatory for muscle function and movements. Most likely such cortical sensory programs are also active in the normal individual in many movements. If for every movement proprioceptic input would be necessary according to the original concept there would be a substantial time delay and many mistakes done. It is therefore likely that - as also has been described in experimental situations - there is a direct sensory - motor cortex interaction depending on an established sensory program in the cortex for rapid motor performances which of course in the normal situation is tuned properly by proprioceptic input particularly in dexterous and fine manipulative tasks.

\section{PRESENT AND FUTURE}

Thus, surgical replantation of avulsed cervical and lumbosacral ventral spinal roots can promote spinal cord or central nervous regeneration of autonomic and somatic motoneurons with functional re-innervation of peripheral targets such as muscles and pelvic organs. Unexpectedly, and as it seems "fringe benefits" from such surgery are pain alleviation as well as central cortical proprioceptive modulation of restored muscle activity. Both central nervous system regeneration and muscle function without proprioceptive function are of conceptual importance, but there is room for improvements to augment functional recovery particularly regarding restoration of sensory function but also motor activity.

Root avulsion injury results in time dependent neuronal loss. Neuroprotective strategies are therefore of interest. The surgery to replant avulsed ventral roots to the spinal cord has a documented effect on motoneuron survival (Bergerot et al., 2004; Bigbee et al., 2008; Eggers et al., 2008). This effect is, however, transient and in long time follow up there is no real difference in motoneuron population after avulsion only and avulsion with root replantation (Bergerot et al., 2004; Eggers et al., 2010). There are, however, some conflicting data on this issue. The amount of motoneurons surviving after avulsion injury was found to be lower in situations where also the dorsal root had been avulsed (Gu et al., 2004) or when neurons expressing ChAT immunohistochemistry were assessed (Hoang et al., 2006). This discrepancy could depend on different surgical techniques but most likely how the motoneuron population was estimated as quantification varies due to the staining method, with immunohistochemistry revealing greater loss than histological dye. Neurotrophic factors as well as pharmacological agents also have a neuroprotective effect. Brain derived neurotrophic factor (BDNF) but in particular glia derived neurotrophic factor (GDNF) has experimentally proved to be effective in motoneuron maintenance. In combinatorial experiments when such substances were given to animals after root avulsion and replantation there was a good persisting effect with improved functional outcome (Bergerot et al., 2004).

Several already approved pharmacological products have been tested experimentally in root avulsion with longitudinal spinal cord injury. One of these, riluzole, which currently is used for amyelotrophic lateral sclerosis, has shown a good neuroprotective effect after root avulsion (Bergerot et al., 2004). Interestingly, a combination of riluzole and GDNF applied after ventral root avulsion and replantation showed a good, robust regeneration. Using two different locomotor tests it was found that significant functional recovery approaching normal values occurred only after re-implantation of avulsed ventral roots together with GDNF and riluzole treatment. These results show that functional recovery is correlated to enhanced dendritic complexity and increased survival 
of motoneurons. Riluzole promotes dendritic outgrowth and neurotrophins such as GDNF promote motoneuron survival and axonal regeneration and both are required for functional recovery possibly by increasing neurotransmitter level or synthesis in motoneurons. Combinations of substances which provoke such neuronal reactions are needed to achieve a good outcome from repair of root avulsion injury. Thus, combination treatment may offer a new therapeutic strategy for treating patients with avulsion injury. However, the delivery of these substances is challenging. For instance a gene therapy approach using adeno-associated viral (AAV) vectors for long time delivery was indeed successful in preventing motoneuron death due to the trophic effect of the secreted neurotrophic factors, but the regenerated axons were "locked up" in the vicinity of the delivery site of the viral vectors and there was no distal growth and consequently no functional restoration. This may be solved by creating a longitudinal gradient of the neurotrophic factors by using a controllable or transient gene product.

However, even if neurons are protected functional recovery is presently limited to proximal regions. This is due to a restricted amount of regrowing axons as well as the slow rate of regeneration leaving distant targets denervated for a long time. Chronically denervated Schwann cells cannot maintain correct neurotrophic support and distal muscles become atrophied and fibrosed with eventually very little intact muscle to innervate (Suliman and Gordon, 2000). In preliminary studies using OEC applied to the site of ventral root implantation there was a four-fold increase in regenerating axons ( $\mathrm{Li}$ et al., 2007). It is of course not yet clear whether the increased number of motor fibers induced by presence of transplanted OECs in the re-implanted ventral root would per se overcome the 'lure' of simply increasing the innervation of the nearer proximal muscles (Eggers et al., 2010).

Strategies should however not only be directed to problems of neuroprotection and augmenting number of regenerating axons. If the pace of regrow cannot be increased means to save denervated muscles should be implemented. There are now preliminary data that already existing drugs could have such effects (Shortland, personal communication)

In spite of the "reserve cortical sensory capacity" which helps in more simple movements as well as in alleviation of pain through corrections of motor trajectories, it is necessary to try to re-establish the afferent loop of the damaged spinal cord connections. Most promising in experimental work has been the application of OEC, a type of stem cells, which has promoted re-entry of primary sensory axons into the dorsal horn of the spinal cord (Li et al., 2004). An interposition of OEC between the Schwann cells and the astrocytes induced "bridging channels" from the PNS to the CNS parts of the root that allowed

\section{REFERENCES}

Bergerot, A., Shortland, P. J., Anand, P., Hunt, S., and Carlstedt, T. (2004). Co-treatment with riluzole and GDNF is necessary for functional recovery after ventral root avulsion injury. Exp. Neurol. 187, 359-366.

Berman, J., Birch, R., and Anand, P. (1998) Pain following human brachial plexus injury with spinal cord root avulsion

dorsal root nerve fibers to enter the spinal cord (Li et al., 2004). This arrangement appears similar to the naive arrangement at the TR of Schwann cell columns surrounding individual nerve fibers in the channels of the astrocytic fringe. Regenerated nerve fibers could be demonstrated within the dorsal horn as well as within the white matter of the dorsal columns. Functional restoration has also been reported with this technique as progressive improvements in paw grasping a frame bar during climbing (Ibrahim et al., 2009).

An alternative method to re-establish sensory connectivity would simply be to discard the primary sensory neuron, i.e., to excise the dorsal root ganglion, and replant the dorsal root into the dorsal horn to allow secondary sensory neurons to extend into the periphery. This was previously demonstrated in animal experiments (Carlstedt, 1985b) and has according to preliminary data with some success recently been applied clinically.

Interest should also be directed to the effect on second and third order sensory neurons from the impact of sensory root avulsion. It has been documented that avulsion rather than rhizotomy leads to about 30\% death of dorsal horn cells (Chew et al., 2008). There is today no information about a transynaptic effect of such trauma which of course is crucial once regeneration of primary sensory neurons into the dorsal horn is established. Maintenance of secondary sensory neurons is obviously also of importance with regards to the classical excruciating avulsion pain which today is poorly understood. Of interest in this context is also the role played by increase in glia activity in the dorsal horn in particular by microglia.

Further achievements have to rely on new basic science methods. For instance with microarray techniques the complicated biological events resulting from gene activity after injury and repair can be assed and valuable insight and cues how to advance would be gained. With regards to curtailed or restricted axonal growth within the spinal cord there are new compounds for neurite outgrowth regulation that are of interest. Some of those are small lipophilic molecules acting as receptor agonists, which potentially can reach injured neurons without difficulties in administration or dosage. For instance, functional regeneration of sensory axons into the spinal cord was achieved after stimulation of the retinoic acid receptor by such compounds (Wong et al., 2006).

With regards to surgery currently most cases treated are from brachial plexus traction injury. A caudal spinal cord injury at conus level has many similarities with the root avulsion as mainly the lower motoneurons and CNS parts of the peripheral sensory neurons together with nervous control over bladder and bowel function is affected. This type of injury will in the future be considered for similar type of surgical strategy in man as for the spinal cord injured patients control over pelvic organs has the highest priority.

Carlstedt, T. (1985). Regenerating axons form nerve terminals at astrocytes. Brain Res. 347, 188-191.

Carlstedt, T. (1985b). Dorsal root innervation of spinal cord neurons after dorsal root implantation into the spinal cord of adult rats. Neurosci. Lett. 55, 343-348.

Carlstedt, T. (2007). Central Nerve Plexus Injury. London: Imperial College Press.
Carlstedt, T., Anand, P., Htut, M., Misra, P., and Svensson, M. (2004). Restoration of hand function and "breathing arm" from spinal cord repair of C5-T1 brachial plexus avulsion injury. Case Report. Neurosurg. Focus 16, 7.

Carlstedt, T., Grane, P., Hallin, R., and Noren, G. (1995). Return of function after spinal cord implantation of 
avulsed spinal nerve roots. Lancet 346 , 1323-1325.

Carlstedt, T., Hultgren, T., Nyman, T., and Hansson, T. (2009). Cortical activity and hand function restoration in a patient after spinal cord surgery. Nat. Rev. Neurol. 5, 571-574.

Chew, D. J., Leinster, V.H.L., Sakthithasan, M., Robson, M. L., Carlstedt, T., and Shortland, P. J. (2008). Cell death after dorsal root injury. Neurosci. Lett. 433, 231-234.

Chong, M. S., Woolf, C. J., Haque, N. S. K., and Anderson, P.N. (1999). Axonal regeneration from injured dorsal roots into the spinal cord of adult rats. J. Comp. Neurol. 410, 42-54.

Cullheim, S., Carlstedt, T., Lindå, H., and Risling, M. (1989). Motoneurons reinnervate skeletal muscle after ventral root implantation into the spinal cord. Neuroscience 29, 725-733.

Eggers, R., Hendriks, W. T. J., Tannemaat, M. R., van Heerikhuize, J.J., Pool, C. W., Carlstedt, T., Zaldumbide, A., Hoeben, R. C., Boer, G. J., and Verhaagen, J. (2008). Neuroregenerative effects of reimplantation of genetically modified roots in a rat model for ventral spinal nerve avulsion. Mol. Cell. Neurosci. 39, 105-117.

Eggers, R., Tannemaat, M. R., Ehlert, E. M., and Verhaagen, J. (2010). A spatio-temporal analysis of motoneuron survival, axonal regeneration and neurotrophic factor expression after lumbar ventral root avulsion and implantation. Exp. Neurol. 223, 207-220.

Gu, H. Y., Chai, H., Zhang, J. Y., Yao, Z. B., Zhou, L. H., Wong, W. M., Bruce, I. C., and Wu, W. T. (2004). Survival, regeneration and functional recovery of motoneurons in adult rats by reimplantation of ventral root following spinal root avulsion. Eur. J. Neurosci. 19, 2123-2131.

Havton, L. A., and Carlstedt, T. (2009). Repair and rehabilitation of plexus and root avulsions in animal models and patients. Curr. Opin. Neurol. 22, 570-574.

He, Z., and Koprivica, V. (2006). The nogo signalling pathway for regeneration block. Ann. Rev. Neurosci. 27, 341-368.

Hoang, T. X., Pikov, V., and Havton, L. A. (2006). Functional reinnervation of the rat lower urinary tract after cauda equina injury and repair. J. Neurosci. 26, 8672-8679.

Htut, M., Misra, V. P., Anand, P., Birch, R., and Carlstedt, T. (2006). Pain phenomena and sensory recovery following brachial plexus avulsion injury and surgical repairs. J. Hand Surg. 31B, 596-605.

Ibrahim, A. G., Kirkwood, P. A., Raisman, G., and Li, Y. (2009). Restoration of hand function in a rat model of repair of brachial plexus injury. Brain 132, 1268-1276.
Kliot, M., Smith, G. M., Siegal, J. D., and Silver, J. (1990). Astrocyte-polymer implants promote regeneration of dorsal root fibers into the adult mammalian spinal cord. Exp. Neurol. 109, 57-69.

Li, Y., Carlstedt, T., Berthold, C.-H., and Raisman, G. (2004). Interaction of transplanted olfactory-ensheathing cells and host astrocytic processes provides a bridge for axons to regenerate across the dorsal root entry zone. Exp. Neurol. 188, 300-308.

Li, Y., Yamamoto, M., Raisman, G., Choi, D., and Carlstedt, T. (2007). An experimental model of ventral root repair showing the beneficial effect of transplanting olfactory ensheathing cells. Neurosurgery 60, 734-740.

Ramer, M. S., Bishop, T., Dockery, P., Mobarak, M. S., O'Leary, D., Fraher, J. P., Priestley, J. V., and McMahon, S. B. (2002). Neurotrophin-3-mediated regeneration and recovery of proprioception following dorsal rhizotomy. Mol. Cell Neurosci. 19, 239-249.

Suliman, O. A., and Gordon, T. (2000). Effects of short- and long-term Schwann cell denervation on peripheral nerve regeneration, myelination and size. Glia 32, 234-246.

Wang, R., King, T., Ossipov, M. H., Rossomando, A. J., Vanderah, T. W., Harvey, P., Cariani, P., Frank, E., Sah, D. W., and Porreca, F. (2008). Persistent restoration of sensory function by immediate or delayed systemic artemin after dorsal root injury. Nat. Neurosci. 11, 488-496.

Wong, L. F., Yip, P. K., Battaglia, A., Grist, J., Corcoran, J., Maden, M., Azzouz, M., Kingsman, S. M., Kingsman, A. J., Mazarakis, N.D., and McMahon, S. B. (2006). Retinoic acid receptor beta 2 promotes functional regeneration of sensory axons in the spinal cord. Nat. Neurosci. 9, 243-250.

Conflict of Interest Statement: The authors declare that the research was conducted in the absence of any commercial or financial relationships that could be construed as a potential conflict of interest.

Received: 15 March 2010; paper pending published: 23 April 2010; accepted: 31 May 2010; published online: 12 July 2010.

Citation: Carlstedt J (2010) Perspectives on the treatment of the longitudinal spinal cord injury. Front. Neur. 1:11. doi: 10.3389/ fneur.2010.00011

This article was submitted to Frontiers in Neurotrauma a specialty of Frontiers in Neurology.

Copyright $\odot 2010$ Carlstedt. This is an open-access article subject to an exclusive license agreement between the authors and the Frontiers Research Foundation, which permits unrestricted use, distribution, and reproduction in any medium, provided the original authors and source are credited. 